\title{
Análisis de un amortiguador de masa sintonizado óptimo en estructuras asimétricas no lineales
}

\author{
Analysis of an optimal tuned mass damper in non-linear asymmetrical structures
}

\section{Gilda Espinoza, Diego Neira y Albert Cifuentes}

Fecha de entrega: 21 de noviembre 2017 Fecha de aceptación: 30 de mayo 2018

Departamento de Ingeniería Civil y Ambiental, Universidad del Bío-Bío, Avenida Collao 1202, Casilla 5-C, Concepción, Chile, gespinoz@ubiobio.cl, diego.neira.villarroel@gmail.com, albcifer@gmail.com

Se analiza el comportamiento de un amortiguador de masa sintonizado (AMS) óptimo ubicado en una estructura asimétrica de un piso con tres ejes resistentes con comportamiento no lineal en dirección de la excitación sísmica. El comportamiento no lineal es modelado a través de la ecuación de Bouc-Wen. Se consideran dos criterios de optimización, el primero consiste en el balance uniforme y reducción de la energía histerética simultáneamente en los tres ejes resistentes no lineales y el segundo se basa en la minimización del daño de la estructura a través de un funcional de daño propuesto, consistente en la media entre la energía histerética normalizada del sistema y el coeficiente de correlación entre el desplazamiento y rotación de la planta, con el objetivo de alcanzar balance torsional. El estudio se realiza desde un punto de vista estocástico estacionario. Se encuentra que la frecuencia óptima del AMS se sintoniza con la frecuencia lineal equivalente del modo predominante. La posición óptima del AMS, para ambos criterios, se encuentra en el borde que en la condición de estructura sin AMS presenta una mayor deformación y energía histerética. Se observa que para el segundo criterio el término del coeficiente de correlación logra valor nulo, observándose balance torsional de la estructura. Por otra parte, el AMS es eficiente en la reducción de deformación y disipación de energía histerética en la estructura, reduciendo más, tanto la razón de energía histerética con respecto a la energía del sistema asimétrico como la deformación de borde, en el plano más cercano a la posición óptima del AMS.

Palabras claves: amortiguador de masa sintonizado, daño estructural, estructura asimétrica, estructura no lineal, optimización
The behaviour of the tuned mass damper (TMD) attached to an asymmetrical structure with three nonlinear plans in the direction of the seismic excitation is analyzed. The non-linear behaviour is modelled through the Bouc-Wen element. Two optimization criteria are considered: the first one consists on achieving simultaneously the uniform balance and reduction of the hysteretic energy in the non-linear plans; and the second is based on the minimization of the structural damage by means of a proposed damage functional, consisting in the mean between the standardized hysteretic energy of the system and the correlation coefficient between the movement and the rotation of the plant, in order to reach the torsion balance. The study is performed from a stochastic stationary point of view. It is found that the optimal frequency of the TMD is tuned with the linear equivalent frequency to the predominant mode. The optimal position of the TMD, for both studied criteria, is at the border where the greater deformation and hysteretic energy occur on the structure without TMD. It is noted that for the second criterion the term of the correlation coefficient reaches a null value, observing torsion balance of the structure. Also, the TMD is efficient in the reduction of the deformation and hysteretic energy dissipation, reducing more the hysteretic energy ratio with respect to the energy of the asymmetrical system, and the border deformation on the floor plan closer to the optimal position of the TMD.

Keywords: tuned mass damper, structural damage, asymmetrical structure, non-linear structure, optimization

\section{Introducción}

En países con alta sismicidad como Chile, se producen daños excesivos en elementos estructurales y no estructurales debido a excitaciones sísmica intensas. Es 
por esto que se han investigado distintas propuestas de solución. Una de ellas son los amortiguadores de masa sintonizados (AMS). El AMS consiste en una masa secundaria unida a la estructura mediante un amortiguador y un elemento elástico. Las primeras investigaciones sobre los AMS tuvieron como objetivo determinar óptimamente la relación de frecuencias entre el AMS y el sistema principal y el factor de amortiguamiento para un sistema de un grado de libertad no amortiguado (Den Hartog, 1947). Posterior a esto, se investigó el efecto de la inclusión del amortiguamiento en el sistema principal y se determinó parámetros óptimos ante distintas solicitaciones dinámicas. Investigaciones posteriores se orientaron al análisis de la eficiencia del AMS en el control de vibraciones en estructuras debidas a vientos (Kwok y Samali, 1995). De estas se concluye que si la frecuencia natural del AMS es sintonizada con el modo fundamental de la estructura principal casi toda la energía de vibración de la estructura principal es transferida al AMS y es disipada por el amortiguamiento.

Posteriormente, se investigó acerca de la eficiencia del AMS en el control de estructuras sometidas a excitaciones sísmicas en estructuras lineales. Entre estas investigaciones se encuentra a Villaverde (1994) quien analizó tres estructuras diferentes, un edificio de corte de dos pisos en 2D (dos dimensiones), una construcción en base a marcos de un piso en 3D (tres dimensiones) y la tercera, un puente atirantado en 3D usando nueve tipos diferentes de registros sísmicos. Los resultados numéricos y experimentales mostraron que la eficiencia del AMS en reducir la respuesta de la misma estructura durante diferentes eventos sísmicos, o diferentes estructuras durante el mismo evento sísmico es significativamente diferente; algunos casos obtuvieron buen desempeño y en otros obtuvieron poco o incluso nulo efecto. Esto implica que hay una dependencia de la reducción alcanzada con las características del movimiento sísmico que excita la estructura.

Estudios posteriores analizan el comportamiento del AMS cuando la estructura principal posee un comportamiento no lineal. Una de estas investigaciones estudia la influencia de la intensidad del movimiento en la efectividad del AMS para reducir el daño en una estructura no lineal (Soto-Brito y Ruiz, 1999). Los resultados mostraron que la instalación de un AMS en una estructura no lineal puede no reducir el desplazamiento máximo bajo sismos severos, pero sí puede ser exitoso en sismos de mediana y baja intensidad. Sin embargo, la reducción del desplazamiento máximo en estructuras no lineales puede ser un criterio de evaluación insuficiente ya que no toma en cuenta el efecto del daño acumulativo en la estructura producido por los ciclos de fatiga.

Wong (2008) estudia la capacidad para disipar energía de una estructura inelástica con un AMS, los resultados indicaron que un AMS aumenta la eficiencia de disipación de energía de la estructura con lo que el daño que sufre ante un sismo se ve reducido. Posteriormente se obtuvieron los parámetros óptimos de un AMS para la protección de estructuras no lineales sometidas a un input sísmico con un enfoque estocástico (Sgobba y Marano, 2010). Se estudiaron tres funciones objetivo: la desviación estándar del desplazamiento de la estructura del sistema protegido en comparación con uno desprotegido, el valor promedio de la energía histerética disipada por la estructura con AMS respecto a la misma sin AMS y un funcional asociado a los dos índices anteriores, considerando un modelo histerético Bouc-Wen (Wen, 1976). Zhang y Balendra (2013) concluyen que cuando se considera no linealidad de la estructura, un diseño óptimo puede producir una mayor reducción de daño comparado con fórmulas de diseño basadas en una respuesta elásticas. Estas investigaciones se realizan bajo una hipótesis de estructuras simétricas en planta, lo que en gran medida por restricciones arquitectónicas no es posible, lo que genera la necesidad de ampliar la investigación hacia estructuras asimétricas.

Se han realizado varias investigaciones acerca del comportamiento lateral-torsional de una estructura asimétrica, con respecto a la influencia de la excentricidad. Dentro de ellas se encuentra el estudio de BenaventeCliment et al. (2014), quienes estudian una estructura asimétrica escalada de hormigón, la cual muestra que existe no linealidad al ser sometida a una excitación en una mesa vibradora si se analiza la incertidumbre de los procesos constructivos. Lin et al. (2016) proponen un factor de amplificación torsional para controlar el efecto torsional en las estructuras como una alternativa al control de torsión desde el punto de vista del diseño sismorresistente tradicional. El primer estudio se enfocó a la obtención de los parámetros óptimos para minimizar la razón entre la 
raíz media cuadrática del desplazamiento y la rotación del sistema con AMS con respecto al sistema sin AMS (Jangid y Datta, 1997). Este estudio concluyó que la efectividad de múltiples AMS en controlar la respuesta lateral de un sistema torsionalmente acoplado decrece con el grado de asimetría. Posteriormente se obtuvo los parámetros óptimos de diseño de dos AMS en edificios asimétricos de múltiples pisos bajo una excitación sísmica bidireccional, utilizando como criterio de optimización la minimización de la media cuadrática de desplazamiento asociado al modo dominante (Lin et al., 2000). Como otro criterio también se propuso maximizar diferentes funciones de reducción de respuesta de interés, tales como corte basal, aceleraciones de piso y drift de entre piso para cuatro AMS (Singh et al., 2002). También se estudió la minimización del cociente entre la media cuadrática de desplazamiento del grado de libertad en el control de la estructura con y sin MAMS (múltiples AMS) (Ueng et al., 2008). Posteriormente, se propuso el concepto de balance torsional de una estructura asimétrica de un piso con un AMS obteniendo la posición y frecuencia óptimas del AMS que permite reducir el desplazamiento en los bordes y la correlación entre el desplazamiento y el giro medido en el centro geométrico de la planta considerando procesos aleatorios de ancho de banda amplio y angosto (Almazán et al., 2012). Sin embargo, aún no se realizan investigaciones sobre estructuras asimétricas no lineales, con enfoque estocástico de la excitación sísmica, con el objetivo de evaluar el comportamiento del AMS.

Esta investigación propone dos nuevos criterios de reducción del daño sobre una estructura asimétrica no lineal, el primer criterio asociado a la energía histerética disipada, término asociado directamente al daño estructural, y que consiste en reducir la energía histerética disipada por los planos resistentes de una estructura asimétrica no lineal, teniendo como objetivo disminuir y alcanzar el mismo daño en todos los planos simultáneamente, y el segundo criterio consiste en la minimización del daño en la estructura asimétrica no lineal, medido a través de un funcional de daño que considera, la energía histerética disipada en la estructura y la reducción de las demandas de deformación de bordes de la planta, por medio de la reducción de un coeficiente de correlación, entre desplazamiento y rotación de planta.

\section{Modelo estructural}

En esta investigación se ha estudiado una estructura monosimétrica de un piso sometida a un movimiento sísmico unidireccional, a la cual se le ha incorporado un AMS lineal. La planta tiene forma rectangular de largo $a$ y ancho $b(a / b=4)$, con masa traslacional $m_{s}$, radio de giro $\rho=\sqrt{\left(a^{2}+b^{2}\right) / 12}$ y formado por tres ejes resistentes no lineales en la dirección del movimiento sísmico. Por lo tanto, su centro geométrico CG coincide con su centro de rigidez $\mathrm{CR}$, mientras que su centro de masa $\mathrm{CM}$ se ubica en el eje $X$ a una distancia $e_{x}$ (excentricidad estática) del CR (ver Figura 1). El periodo lateral no acoplado en dirección $Y$ de la estructura principal sin AMS es $T_{s y}$. Para la razón de rigidez torsional $\left(\Omega_{\mathrm{s}}\right)$ se utilizaron valores de 0.7 , 1.0 y 1.3 , para estudiar el efecto del AMS en estructuras torsionalmente flexibles, híbridas y rígidas. Además se definió un amortiguamiento modal $\xi_{\mathrm{s}}$ igual a 0.05 en todos los modos de vibración para la estructura sin AMS. Por su parte, el AMS es considerado como un dispositivo lineal tipo Kelvin, localizado en el eje $X$ a una distancia $p_{x}$ del CR. Su masa total es asumida como el $2 \%$ de la masa traslacional total de la estructura principal.

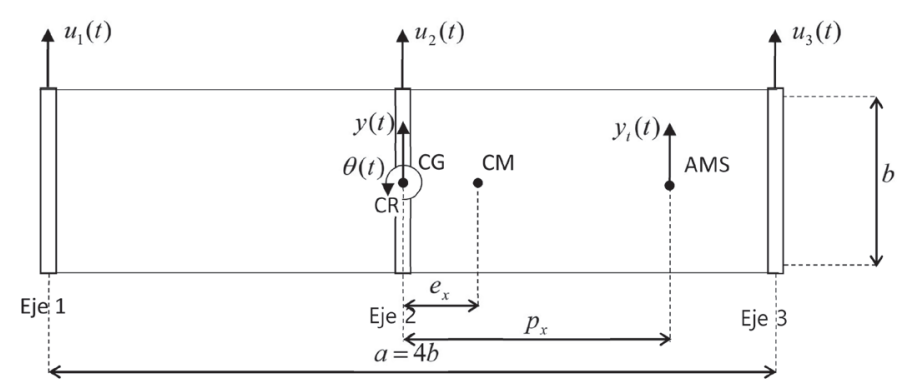

Figura 1: Planta de modelo estructural monosimétrico no lineal con un AMS

\section{Relación constitutiva}

El modelo clásico de Bouc-Wen BW (Bouc, 1967, 1969; Wen, 1976) ha sido ampliamente utilizado en análisis dinámicos determinísticos y estocásticos de estructuras reales con una precisión razonable, tomando en cuenta la cantidad de energía disipada con precisión razonable y de acuerdo al movimiento de la estructura. Por esta razón los ejes resistentes de la estructura se modelan mediante elementos no lineales de BW. La fuerza de restauración $f_{n l}$ está dada por la siguiente expresión: 


$$
f_{n l j}=\alpha_{s j} k_{j} u_{j}+\left(1-\alpha_{s j}\right) F_{y j} z_{j} \quad j=1: 3
$$

donde $F_{y}$ es la fuerza de fluencia de la estructura, $u$ es el desplazamiento relativo del eje respecto al suelo, $\alpha_{s}$ es la relación entre la rigidez post fluencia $k_{p i}$ y la rigidez elástica $k_{i}$.

$$
\alpha_{s j}=\frac{k_{p i}}{k_{i}} \quad 0<\alpha_{s j}<1 \quad j=1: 3
$$

Por su parte $z_{j}$ es la variable histerética adimensional del modelo BW, que satisface la siguiente ecuación diferencial de primer orden:

$$
\dot{z}_{j}=\frac{1}{\Delta Y_{j}} \dot{u}_{j}\left(\lambda-\left|z_{j}\right|^{\eta}\left(\beta+\gamma \operatorname{sgn}\left(z_{j}\right) \operatorname{sgn}\left(\dot{u}_{j}\right)\right)\right) \quad j=1: 3
$$

donde $\gamma$ y $\beta$ son parámetros adimensionales que regulan la forma del ciclo histerético, $\lambda$ es el factor de escala general y $\eta$ es el parámetro que controla la suavidad de la transición entre la región lineal y no lineal.

\section{Ecuaciones del movimiento \\ Cinemáticas}

El vector de grados de libertad de la estructura con AMS se define como:

$$
\mathbf{q}(\mathbf{t})=\left[\begin{array}{lll}
y(t) & \theta(t) & y_{t}(t)
\end{array}\right]^{T}
$$

donde $y(t)$ es el desplazamiento lateral del CG en dirección $Y, \theta(t)$ es la rotación de la planta e $y_{t}(t)$ es el desplazamiento del AMS respecto del suelo. Por otra parte, la deformación del AMS, $u_{t}$, puede ser expresada mediante la siguiente ecuación:

$$
u_{\mathrm{t}}=\mathbf{L}_{\mathbf{t}} \mathbf{q}
$$

donde $\mathbf{L}_{\mathbf{t}}=\left[\begin{array}{lll}-1 & -p_{x} & 1\end{array}\right]$ es la matriz de transformación cinemática.

\section{Equilibrio dinámico}

La ecuación de movimiento de la estructura no lineal unida a un AMS lineal, sometida a una excitación unidireccional se puede describir como:

$$
\mathbf{M} \ddot{\mathbf{q}}+\mathbf{C} \dot{\mathbf{q}}+\mathbf{L}_{\mathbf{f}}^{\mathbf{T}} \mathbf{f}_{\mathbf{n l}}+\mathbf{L}_{\mathbf{t}}^{\mathbf{T}} f_{\mathrm{t}}=-\mathbf{M R} \ddot{u}_{g}
$$

$\mathbf{M}=\left[\begin{array}{cc}\mathbf{M}_{\mathbf{s}} & \mathbf{0}_{2 \times \mathbf{1}} \\ \mathbf{0}_{1 \mathbf{2} 2} & m_{t}\end{array}\right] \quad \mathbf{M}_{\mathbf{s}}=\mathrm{m}_{\mathrm{s}}\left[\begin{array}{cc}1 & \mathrm{e}_{\mathrm{x}} \\ \mathrm{e}_{\mathrm{x}} & \rho^{2}\end{array}\right]$

$\mathbf{C}=\left[\begin{array}{cc}\mathbf{C}_{\mathbf{s}} & \mathbf{0}_{\mathbf{2} \mathbf{1}} \\ \mathbf{0}_{1 \times 2} & 0\end{array}\right]+\mathbf{L}_{\mathbf{t}}^{\mathbf{T}} c_{t} \mathbf{L}_{\mathbf{t}} ; \quad \mathbf{R}=\left[\begin{array}{lll}1 & 0 & 1\end{array}\right]^{r}$

$\mathbf{L}_{\mathbf{f}}=\left[\begin{array}{ccc}1 & -a / 2 & 0 \\ 1 & 0 & 0 \\ 1 & a / 2 & 0\end{array}\right]$

donde $\ddot{u}_{g}$ representa la aceleración del suelo y $\mathbf{R}$ el vector de incidencia de éste. Por su parte, $\mathbf{M}_{\mathrm{s}} \mathrm{y} \mathbf{C}_{\mathrm{s}}$ corresponden a las matrices de masa y amortiguamiento de la estructura, respectivamente. Por último $f_{\mathrm{t}}$ corresponde a la fuerza de restauración lineal del AMS que se calcula como:

$f_{t}=k_{t} u_{t}$

siendo $k_{t}$ la rigidez del AMS. $\mathbf{C}_{\mathrm{s}}$ es la matriz de amortiguamiento lineal del sistema principal sin AMS, la que depende de las razones de amortiguamiento modal indicadas en el modelo estructural y la inclusión del amortiguamiento del AMS se hace a través de la matriz de transformación cinemática $\mathbf{L}_{\mathbf{t}}$ como indica (8a). Esta matriz ampliada $\mathbf{C}$, contiene el amortiguamiento lineal de la estructura principal más el aporte de amortiguamiento del AMS, aporta las fuerzas dinámicas de amortiguamiento del segundo término de la ecuación de equilibrio dinámico (6) y las fuerzas dinámicas no lineales se encuentran en el tercer término de (6), separadas de las de amortiguamiento anteriormente señaladas.

\section{Excitación considerada}

Se consideraron dos procesos aleatorios, un proceso de Ancho de Banda $\mathrm{AB}$ amplio, derivado de un registro compatible con NCh 2745 (2003) para suelo firme tipo $\mathrm{B}$, y un proceso aleatorio de $\mathrm{AB}$ angosto derivado del registro del sismo de México de 1985, registrado en la dirección Norte-Sur. Para ambos procesos, se muestra su correspondiente Densidad de Potencia Espectral PSD, $S_{g}(\omega)$, ajustada a un filtro pasabanda correspondiente al filtro Kanai-Tajimi modificado por Penzien KTP (Clough y Penzien, 1975):

con, 


$$
S_{g}(\omega)=S_{0} \frac{\omega_{g}^{4}+4 \xi_{g}^{2} \omega_{g}^{2} \omega^{2}}{\left(\omega_{g}^{2}-\omega^{2}\right)+4 \xi_{g}^{2} \omega_{g}^{2} \omega^{2}} \frac{\omega^{4}}{\left(\omega_{f}^{2}-\omega^{2}\right)^{2}+4 \xi_{f}^{2} \omega_{f}^{2} \omega^{2}}
$$

donde $S_{0}, \omega_{g}, \xi_{g}, \omega_{f}$ y $\xi_{f}$ son intensidad del ruido blanco, frecuencia del estrato de suelo, amortiguamiento del estrato de suelo, frecuencia del filtro y amortiguamiento del filtro, respectivamente. Estos parámetros fueron ajustados por mínimos cuadrados y sus valores se muestran en la Tabla 1.

Tabla 1: Parámetros del filtro Kanai-Tajimi modificado para los dos procesos aleatorios utilizados.

\begin{tabular}{|c|c|c|c|c|c|}
\hline \multirow{2}{*}{ Tipo de entrada } & \multicolumn{5}{|c|}{ Parámetros } \\
\cline { 2 - 6 } & $S_{0}$ & $\begin{array}{c}\omega_{g} \\
\mathrm{rad} / \mathrm{s}\end{array}$ & $\xi_{g}$ & $\begin{array}{c}\omega_{f} \\
\mathrm{rad} / \mathrm{s}\end{array}$ & $\xi_{f}$ \\
\hline $\begin{array}{c}\text { Ancho de banda } \\
\text { amplio }\end{array}$ & 1335.6 & 3.05 & 0.041 & 8.48 & 0.90 \\
\hline $\begin{array}{c}\text { Ancho de banda } \\
\text { angosto }\end{array}$ & 207.23 & 16.57 & 0.491 & 3.02 & 0.48 \\
\hline
\end{tabular}

\section{Linealización estadística equivalente}

La linealización estadística equivalente es una de las técnicas más utilizadas para el análisis de sistemas no lineales sometidas a una excitación aleatoria (Baber y Wen, 1981). Utilizando esta técnica (3) puede ser representada de la siguiente forma:

$$
\dot{z}=-K_{e q}\left(\mathbf{V}_{\mathbf{q}^{\prime}} t\right) z-C_{e q}\left(\mathbf{V}_{\mathbf{q}^{\prime}} t\right) \dot{u}
$$

donde $\mathbf{V}_{\mathrm{q}}$ es la matriz de covarianza asociada al vector $\mathbf{q}(\mathbf{t}), \mathrm{y} K_{e q}$ y $C_{e q}$ son coeficientes que dependen de ella y se expresan como:

$$
\begin{gathered}
K_{e q}=\sqrt{\frac{2}{\pi}} \beta\left[\sigma_{\dot{u}}+\frac{\gamma_{\dot{u} z}}{\sigma_{z}}\right] \\
C_{e q}=\sqrt{\frac{2}{\pi}} \beta\left[\sigma_{z}+\frac{\gamma_{\dot{u} z}}{\sigma_{\dot{u}}}\right]-1
\end{gathered}
$$

siendo $\sigma_{\dot{u}}$ y $\sigma_{z}$ las desviaciones estándar de las variables ù y $z$, respectivamente. A su vez $\gamma_{\mathrm{uz}}$ corresponde a la covarianza cruzada entre estas variables. Por su parte $\mathbf{V}_{\mathbf{q}}$ se determina mediante la siguiente ecuación:

$$
\mathbf{V}_{\mathbf{q}}=E\left\{\mathbf{q}(\mathbf{t}) \mathbf{q}(\mathbf{t})^{\mathbf{T}}\right\}=\frac{1}{2 \pi} \int_{-\infty}^{\infty} \mathbf{H}_{\mathbf{q}}(j \omega) S_{g}(\omega) \mathbf{H}_{q}^{*}(j \omega) d \omega
$$

donde $\mathrm{E}\{\}$ corresponde al valor esperado del proceso, $\mathbf{H}_{\mathbf{q}}(j \omega)$ se define como la matriz de respuesta en frecuencia y $\mathbf{H}_{\mathbf{q}}^{*}(j \omega)$ es su matriz transpuesta conjugada.

\section{Energía histerética}

El daño en estructuras sujetas a movimientos sísmicos es ocasionado por el desplazamiento de la estructura y por el efecto de la fatiga que experimenta el material. Este último efecto está relacionado con la cantidad de energía histerética que la estructura disipe en el tiempo que dure el movimiento. Por lo tanto, se correlaciona el término de la energía histerética disipada $e_{h}(t)$ con el daño de la estructura. Este se puede obtener a partir de la siguiente expresión:

$$
\mathrm{e}_{\mathrm{h}}(t)=\frac{\mathrm{E}}{\mathrm{m}}=\left(1-\alpha_{\mathrm{s}}\right) \omega_{\mathrm{s}}^{2} \int_{0}^{\mathrm{t}} \mathrm{z}(\tau) \dot{\mathrm{u}}(\tau) \mathrm{d} \tau
$$

siendo el valor promedio:

$$
\left\langle e_{h}\right\rangle=\left(1-\alpha_{s}\right) \omega_{s}^{2} \int_{0}^{T} \gamma_{\dot{u} z}(\tau) d \tau=\left(1-\alpha_{s}\right) \omega_{s}^{2} \gamma_{\dot{u} z} T
$$

donde $T$ es el tiempo de duración del proceso de excitación considerado, $\gamma_{\text {ùz }}$ es la covarianza cruzada entre el desplazamiento del elemento no lineal y la variable histerética $z$ del ciclo histerético de BW. Dado que este estudio se basa en un análisis estocástico, el valor promedio de la energía histerética en el sentido probabilístico de un proceso estacionario es el que se expresa en (17) siguiendo lo realizado por Sgobba y Marano (2010).

\section{Criterios de optimización}

En este trabajo se proponen dos funcionales de optimización, como criterios de control de daño para estructuras con comportamiento no lineal controlados por un AMS optimizados.

\section{Criterio 1, C1}

El problema de optimización consiste en minimizar el máximo del daño en la estructura en todos los planos en forma simultánea.

$\mathrm{F}=\left\{\begin{array}{l}\left(1-\alpha_{s 1}\right) \omega_{\mathrm{s}}^{2} \gamma_{\dot{\mathrm{u}} \mathrm{z} 1} \mathrm{~T} \\ \left(1-\alpha_{s 2}\right) \omega_{\mathrm{s}}^{2} \gamma_{\mathrm{u} z 2} \mathrm{~T} \\ \left(1-\alpha_{s 3}\right) \omega_{\mathrm{s}}^{2} \gamma_{\dot{\mathrm{u}} \mathrm{z}} \mathrm{T}\end{array}\right\}$ 
Minimizar $J_{l}\left(\omega_{d}, p_{x}, \xi_{d}\right)=\operatorname{Max}(\mathbf{F})$

Sujeto a: $\quad-\frac{a}{2}<p_{x}<\frac{a}{2}$

\section{Criterio 2, C2}

El segundo criterio de optimización consiste en minimizar un funcional de daño. La variable asociada a la estimación de los máximos desplazamientos de este índice, es el valor absoluto del coeficiente de correlación, entre la rotación y los desplazamientos en planta medidos en el centro geométrico. La variable asociada a la energía histerética es obtenida de la razón de la energía histerética disipada por la estructura asimétrica con un AMS en sus tres planos resistentes con respecto a la energía histerética disipada por una estructura, de configuración similar a la del modelo, pero de excentricidad nula y sometida al mismo input sísmico.

$F_{\text {daño }}=0.5 a b s\left(\rho_{u \theta}\right)+0.5\left(\frac{\mathrm{e}_{\mathrm{h}}}{\mathrm{e}_{\mathrm{h} \sin \text { Ams }}^{0}}\right)$

donde $\rho_{u \varphi}$ es un coeficiente de correlación entre el desplazamiento medido en el centro geométrico de la planta y el giro, $e_{h}$ es la energía histerética de los tres ejes resistentes no lineales del sistema con AMS, $e_{\mathrm{h} \sin \text { Ams }}^{\mathrm{o}}$ es la energía histerética de los los tres ejes resistentes no lineales del sistema simétrico sin AMS y abs es el valor absoluto del coeficiente de correlación.

$$
\begin{array}{ll}
\text { Minimizar } & J_{l}\left(\omega_{d}, p_{x}, \xi_{d}\right)=\operatorname{Max}\left(F_{\text {daño }}\right) \\
\text { Sujeto a: } & -\frac{a}{2}<p_{x}<\frac{a}{2}
\end{array}
$$

\section{Resultados}

\section{Análisis de sensibilidad}

Previo al proceso de optimización, se realizó un análisis de sensibilidad con la finalidad de identificar como influían los parámetros del AMS en los funcionales a estudiar. Para esto se graficaron las curvas de nivel de los dos funcionales de optimización $J\left(\omega_{\mathrm{d}}, p_{x}, \xi_{d}\right)$ para ambos criterios. La Figura 2 muestra las curvas de nivel del funcional 1, C1, mientras la Figura 3 presenta el funcional 2, C2, ambas para una estructura con parámetros $T_{s}=2.5 \mathrm{~s}, e_{x} / \rho=0.3$, $\xi_{s}=0.05, \Omega_{s}=[0.7,1.0,1.3], \mu=0.02, R=5, n=1 \mathrm{y}$ $\alpha_{1}=\alpha_{2}=\alpha_{3}=0.5$ y un proceso de AB amplio. En las Figuras 2 y 3 la fila superior muestra las curvas para $\xi_{d}=\xi_{d}^{(o p t)}$, la fila media para $\omega_{d}=\omega_{d}^{(o p t)}$ y la tercera para $\mathrm{p}_{x}=\mathrm{p}_{x}^{(o p t)}$. Se

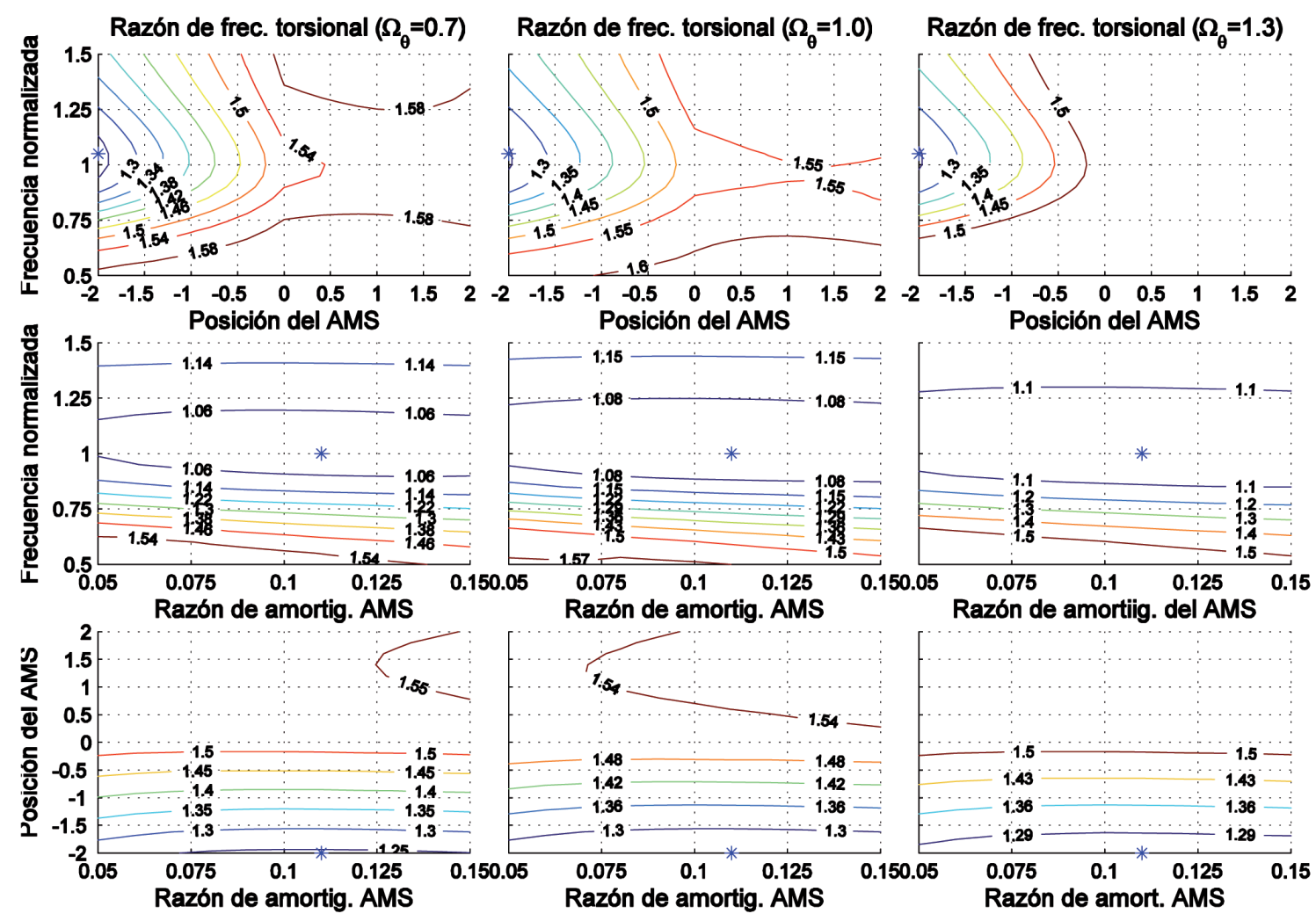

Figura 2: Curvas de nivel del funcional $J$ del criterio 1 para la estructura sometida a un proceso de $\mathrm{AB}$ amplio con $T_{s}=2.5 \mathrm{~s}, e_{x} / \rho=$ $0.3, \xi_{s}=0.05\left(\mu=0.02, R=5, n=1\right.$ y $\left.\alpha_{l}=\alpha_{2}=\alpha_{3}=0.5\right)$ 

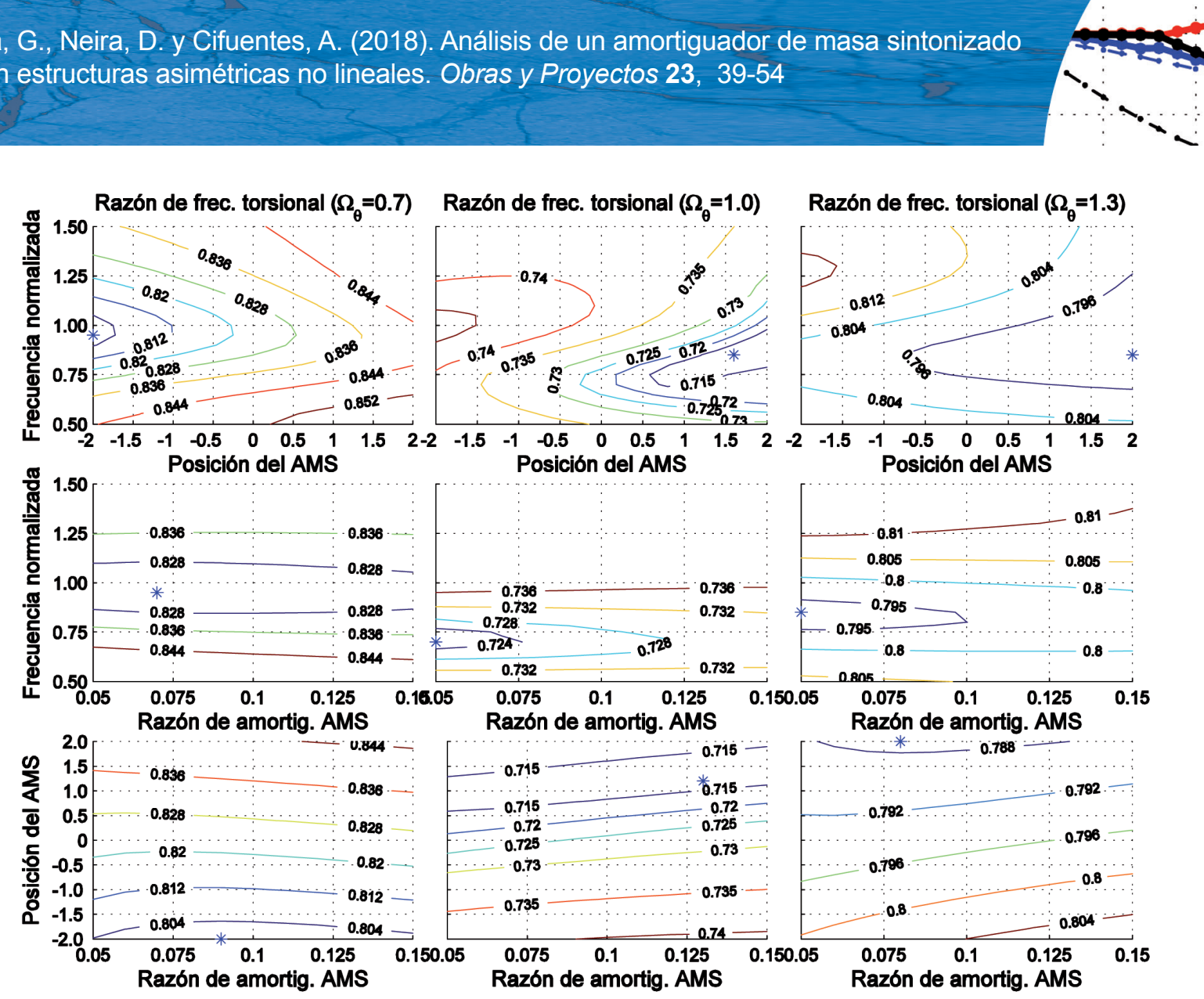

Figura 3: Curvas de nivel del funcional $J$ del criterio 2 para la estructura sometida a un proceso de AB amplio con $T_{s}=2.5 \mathrm{~s}, e_{x} / \rho=$ $0.3, \xi_{s}=0.05\left(\mu=0.02, R=5, n=1\right.$ y $\left.\alpha_{1}=\alpha_{2}=\alpha_{3}=0.5\right)$
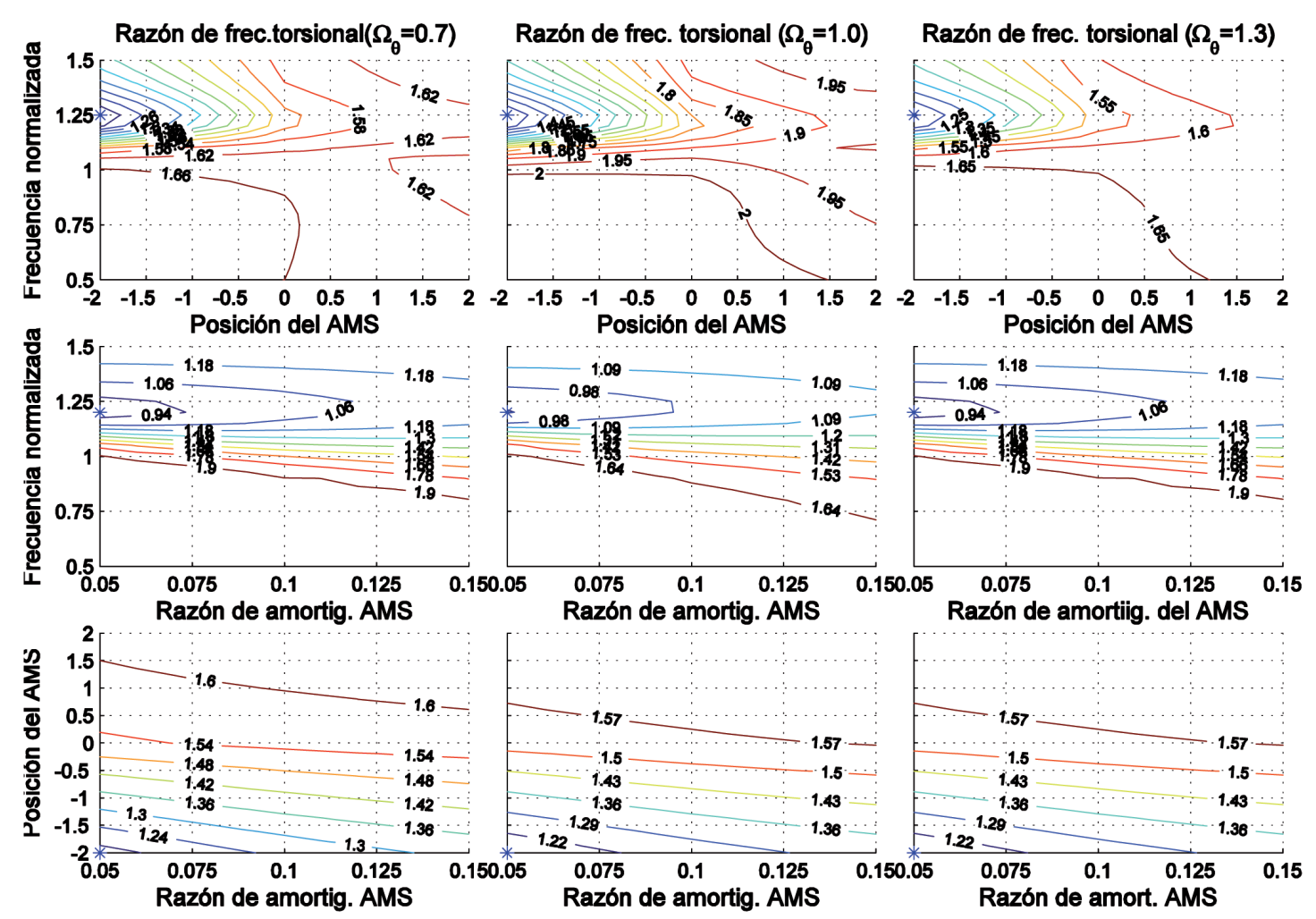

Figura 4: Curvas de nivel del funcional $J$ del criterio 1 para la estructura sometida a un proceso de AB angosto con $T_{s}=2.5 \mathrm{~s}, e_{x} / \rho=$ $0.3, \xi_{s}=0.05\left(\mu=0.02, R=5, n=1\right.$ y $\left.\alpha_{1}=\alpha_{2}=\alpha_{3}=0.5\right)$ 


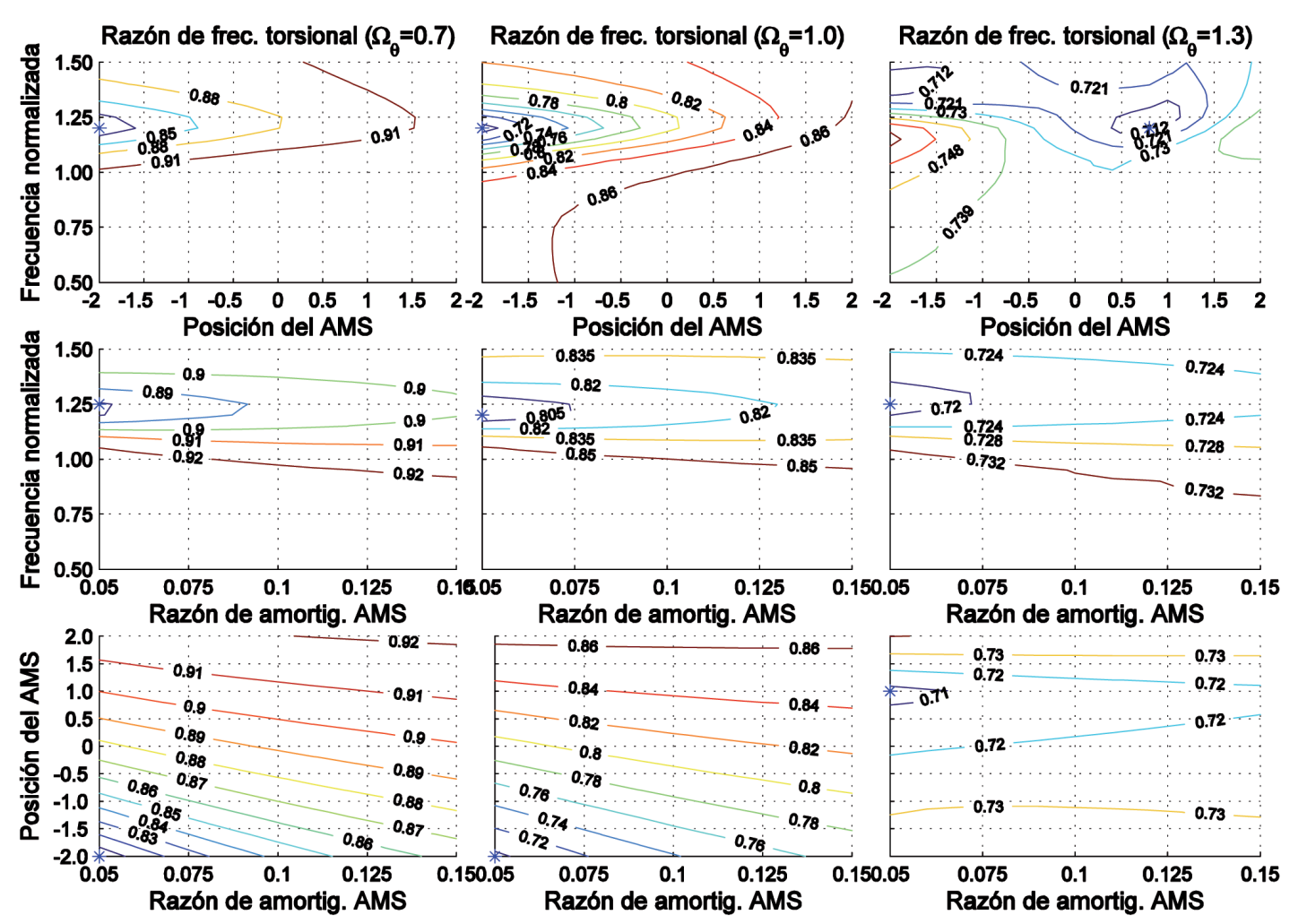

Figura 5: Curvas de nivel del funcional J del criterio 2, para la estructura sometida a un proceso de AB angosto con $T_{s}=2.5 \mathrm{~s}, e_{x} / \rho$ $=0.3, \xi_{s}=0.05\left(\mu=0.02, R=5, n=1\right.$ y $\left.\alpha_{1}=\alpha_{2}=\alpha_{3}=0.5\right)$

observa que en los dos criterios la frecuencia normalizada y la posición del AMS muestran una cierta insensibilidad del funcional con respecto al amortiguamiento del AMS.

La Figura 4 muestra las curvas de nivel del funcional 1, $\mathrm{C} 1$, mientras la Figura 5 presenta el funcional 2, C2, ambas para una estructura con parámetros $T_{s}=2.5 \mathrm{~s}, e_{x} / \rho=0.3, \xi_{s}$ $=0.05, \Omega_{s}=[0.7,1.0,1.3], \mu=0.02, R=5, n=1 \quad \mathrm{y} \alpha_{1}=$ $\alpha_{2}=\alpha_{3}=0.5$ para un proceso de AB angosto. Las Figuras 4 y 5 poseen una distribución análoga a la Figura 2. El funcional resulta ser muy insensible al valor de la razón de amortiguamiento que posee el AMS, tanto para $\mathrm{C} 1$ como para $\mathrm{C} 2$. Esto se traduce en que una variación de la razón de amortiguamiento no genera un cambio en la frecuencia ni posición óptima del AMS. Por esta razón se deja afuera esta variable y se le asigna un valor constante igual a $\xi_{d}=$ 0.12 . Esta elección se basa en consideraciones prácticas de máximo desplazamiento que pueda alcanzar en la realidad un AMS.

\section{Parámetros óptimos del AMS}

Las Figuras 6 y 7 muestran la frecuencia y posición óptima para estructuras con parámetros $T_{s}=1.5 \mathrm{~s}, \xi_{s}=0.05, \Omega_{s}$
$=[0.7,1.0,1.3], \mu=0.02, R=5, n=1 \quad$ y $\quad \alpha_{1}=\alpha_{2}=\alpha_{3}$ $=0.5$, sometidas a un proceso de AB amplio (Figura 6) y $\mathrm{AB}$ angosto (Figura 7). En la fila superior se muestran los resultados para la frecuencia óptima FO del AMS normalizada por $\omega_{\mathrm{s}} \mathrm{y}$ las frecuencias naturales lineales equivalentes FLE de la estructura sin AMS. En la segunda fila se grafica la posición óptima del AMS normalizada por el largo, $p_{x}^{(o p t) / a}$. Los valores negativos se miden desde el centro al eje resistente 1 (borde rígido) y los valores positivos desde el centro al eje resistente 3 (borde flexible).

Se observa que los parámetros óptimos obtenidos para ambos criterios siguen un comportamiento similar. En procesos de $\mathrm{AB}$ amplio la razón de frecuencias óptimas del AMS, en estructuras torsionalmente flexibles, tiende a sintonizarse con la frecuencia lineal equivalente más rígida del sistema sin AMS. En cambio, para sistemas torsionalmente híbridos y rígidos tiende a sintonizar con la primera frecuencia lineal equivalente sin AMS. Para procesos de bajo contenido de frecuencias la razón de frecuencias óptimas del AMS se sintoniza para todo el rango de excentricidades con la frecuencia predominante de la excitación $\omega_{\mathrm{i}}=\pi(\mathrm{rad} / \mathrm{s})\left(T_{\mathrm{i}}=2 \mathrm{~s}\right)$. En este caso 

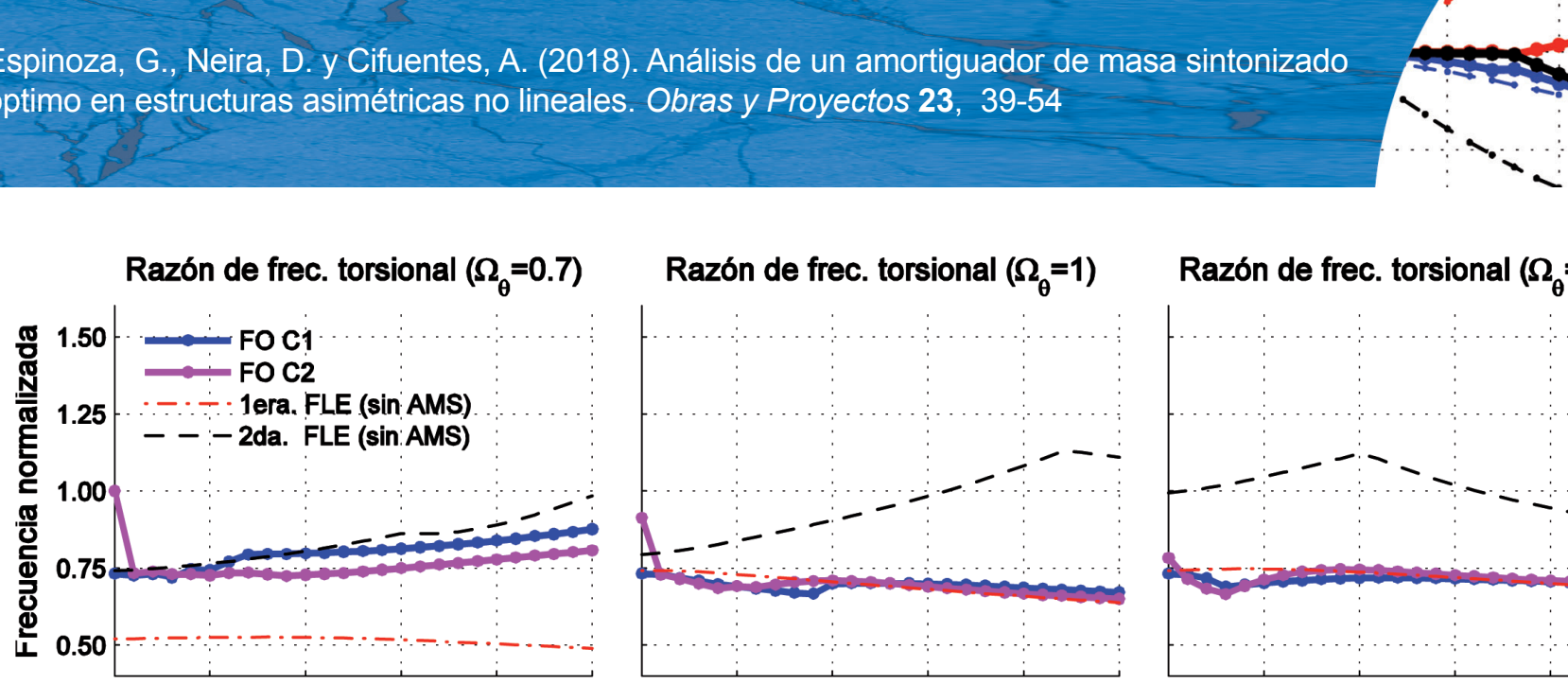

Razón de frec. torsional $\left(\Omega_{\theta}=1.3\right)$
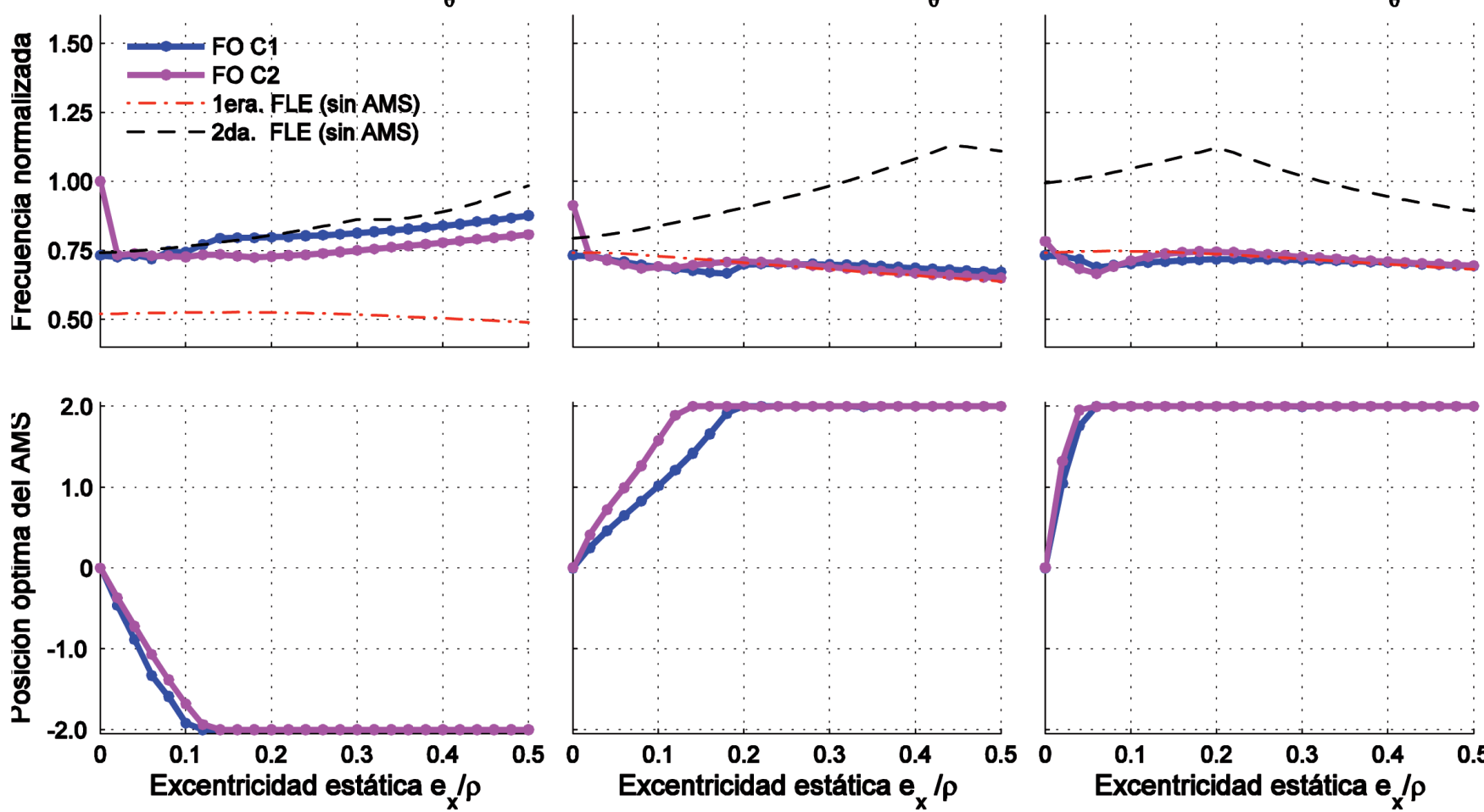

Figura 6: Frecuencia y posición óptimas del AMS según criterios $\mathrm{C} 1 \mathrm{y} \mathrm{C} 2, \mathrm{AB}$ amplio $T_{s}=1.5 \mathrm{~s}, \xi_{\mathrm{s}}=0.05\left(\mu=0.02, e_{\mathrm{s}} / \rho=0.3, n=\right.$ 1 y $\left.\alpha_{1}=\alpha_{2}=\alpha_{3}=0.5\right)$.

Razón de frec. torsional $\left(\Omega_{\theta}=0.7\right)$
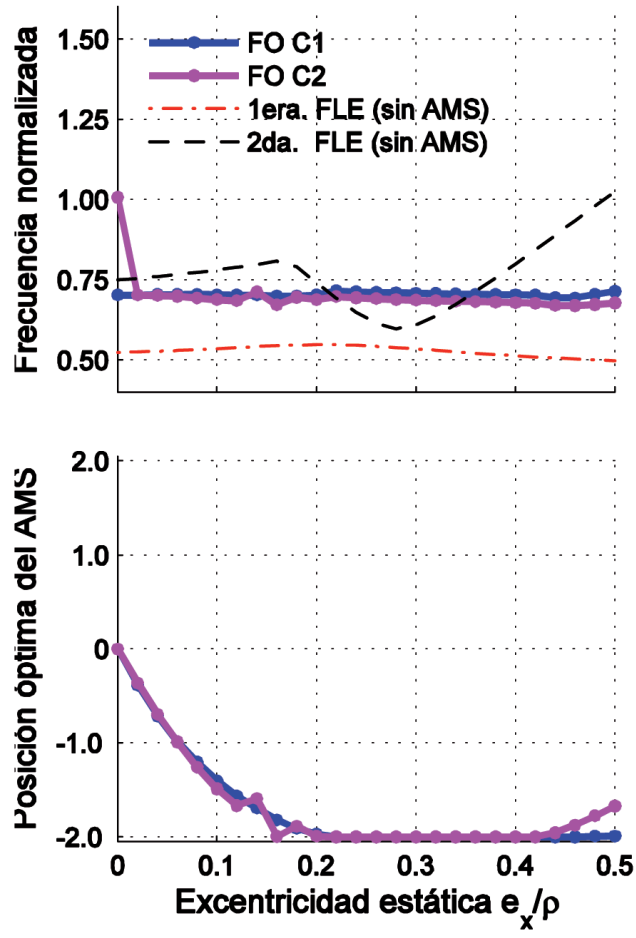
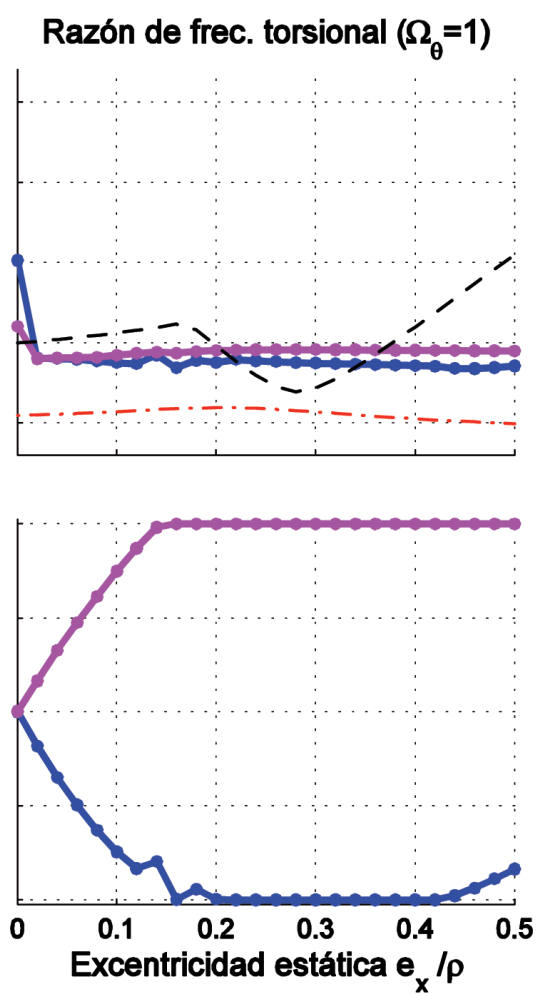

Razón de frec. torsional $\left(\Omega_{\theta}=1.3\right)$
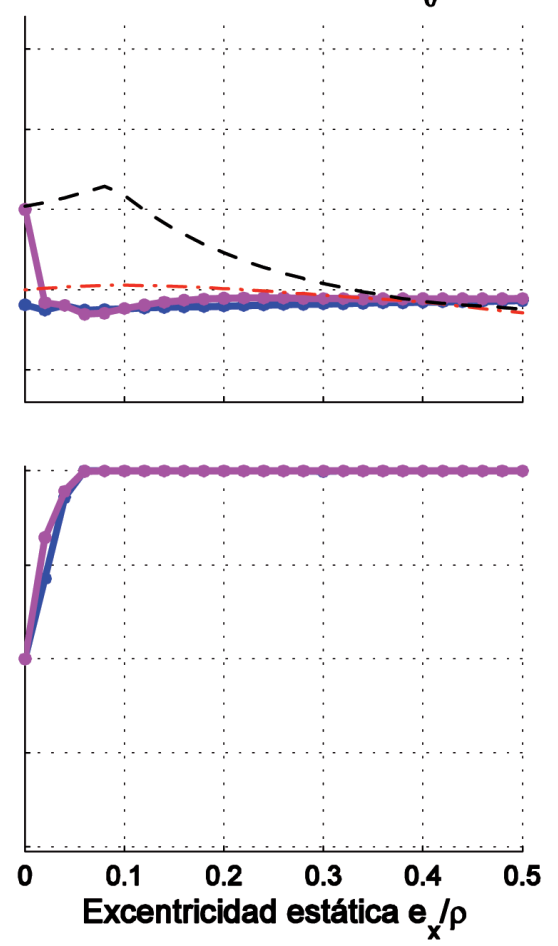

Figura 7: Frecuencia y posición óptimas del AMS según criterios $\mathrm{C} 1$ y $\mathrm{C} 2$, $\mathrm{AB}$ angosto $T_{s}=1.5 \mathrm{~s}, \xi_{\mathrm{s}}=0.05\left(\mu=0.02, e_{\mathrm{s}} / \rho=0.3, n\right.$ $=1$ y $\alpha_{1}=\alpha_{2}=\alpha_{3}=0.5$ ). 
corresponde a la predominante en el sismo de México de 1985, desde el cual se derivó la densidad de potencia espectral, utilizada en la obtención de la matriz de covarianza. Es por este motivo que el AMS se sintoniza en 1.5/2, lo que da la razón de frecuencias 0.75 que adopta como razón de frecuencias óptima para todo el rango de excentricidades para el proceso de $\mathrm{AB}$ considerado. En cuanto a la posición óptima del AMS, en las Figuras 6 y 7 se observa claramente que para ambos criterios y para ambos contenidos de frecuencia, se encuentra en el borde rígido para sistemas torsionalmente flexibles y en el borde flexible para sistemas torsionalmente híbridos y rígidos.

En las Figuras 8 y 9 se analiza el comportamiento de los parámetros óptimos a medida que la estructura incursiona en el rango no lineal. Para esto se grafica la frecuencia y posición óptimas del AMS en función del factor de reducción $R$. Al igual que los gráficos anteriores en la primera fila se muestran los resultados para la frecuencia óptima FO del AMS normalizada por $\omega_{\mathrm{s}}$ y las frecuencias naturales lineales equivalentes FLE de la estructura sin AMS. En la segunda fila se grafica la posición óptima del TMD normalizada por el largo, $p_{x}^{(o p t) / a}$. Estos valores fueron obtenidos para una estructura con parámetros $T_{s}=$ $1.5 \mathrm{~s}, \xi_{s}=0.05, \Omega_{s}=[0.7,1.0,1.3], \mu=0.02, \mathrm{e}_{\mathrm{x}} / \rho=0.3, R$ $=5, n=1$ у $\alpha_{1}=\alpha_{2}=\alpha_{3}=0.5$. Los resultados muestran que a medida que aumenta el factor de reducción, en procesos de ancho de banda amplio, la frecuencia se va desintonizando a medida que la estructura va haciéndose más no lineal para todas las razones de frecuencia torsional. En cambio, para procesos de ancho de banda angosto, la razón de frecuencias es independiente de la no linealidad de la estructura y se sintoniza con la frecuencia predominante del input. En cuanto a la posición óptima, se observa que ésta es insensible al grado de no linealidad de la estructura y se ubica en el borde rígido para estructuras torsionalmente flexibles y en el borde flexible para estructuras torsionalmente híbridas y rígidas, de acuerdo a lo observado para una excentricidad normalizada de 0.3 .

Las Figuras 10 y 11 muestran en la primera fila la energía histerética disipada por cada plano resistente, con y sin AMS, normalizada por la energía del sistema simétrico. En la segunda fila se entrega la desviación estándar de la deformación de los bordes, con y sin AMS, normalizada por la deformación del sistema simétrico, para el proceso
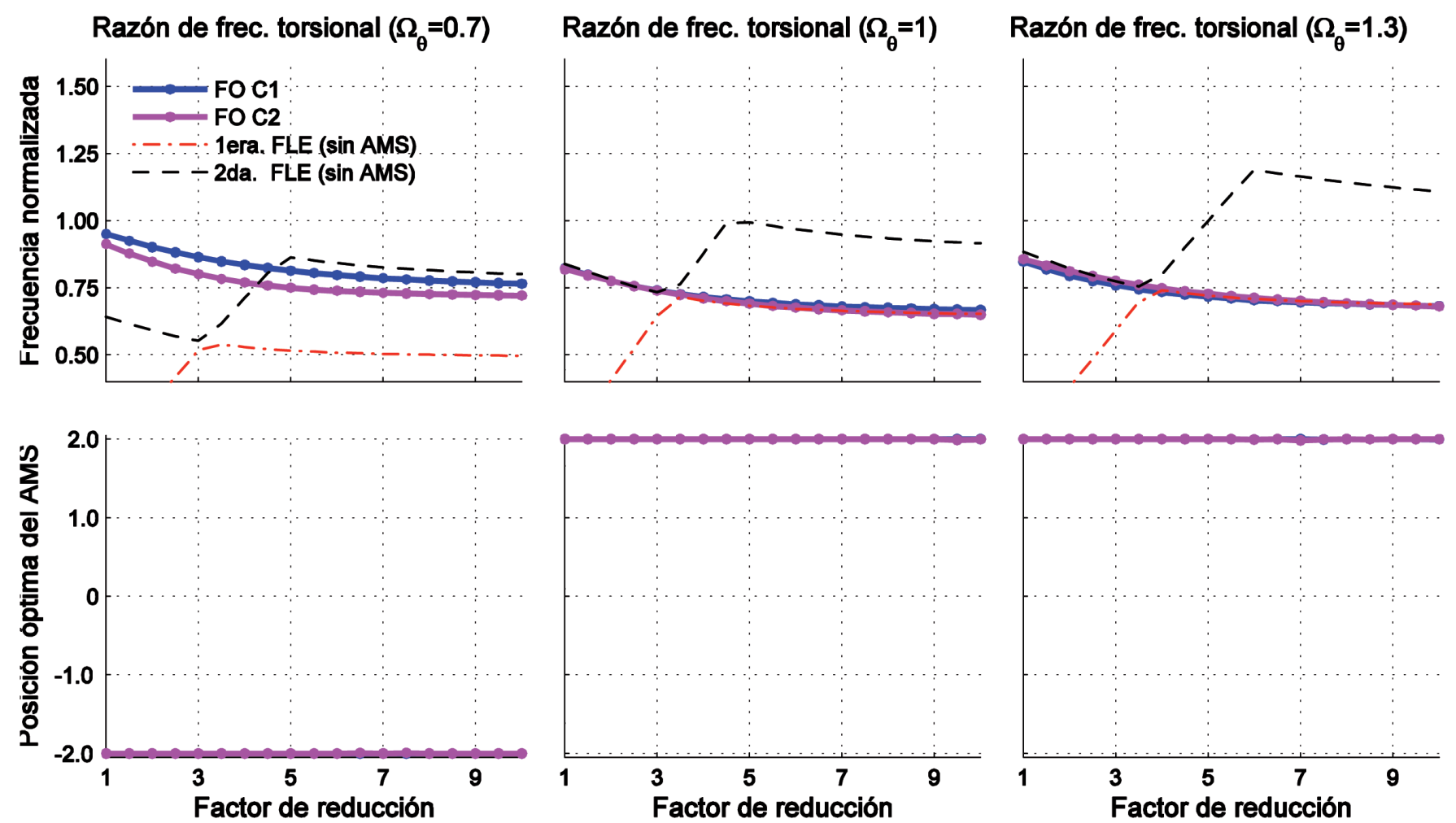

Figura 8: Frecuencia y posición óptimas del AMS según criterios C1 y C2, AB amplio $\left(T_{s}=1.5 \mathrm{~s}, \xi_{\mathrm{s}}=0.05, \mu=0.02, e_{\mathrm{s}} / \rho=0.3, n\right.$ $=1$ y $\left.\alpha_{1}=\alpha_{2}=\alpha_{3}=0.5\right)$. 


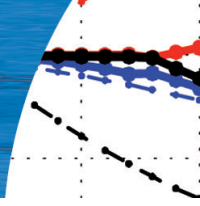

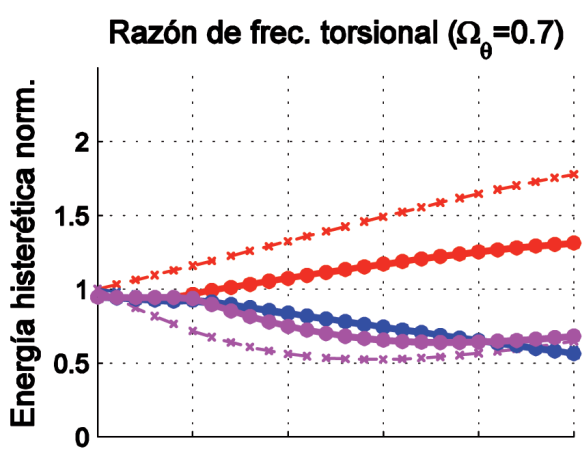

Razón de frec. torsional $\left(\Omega_{\theta}=1\right)$
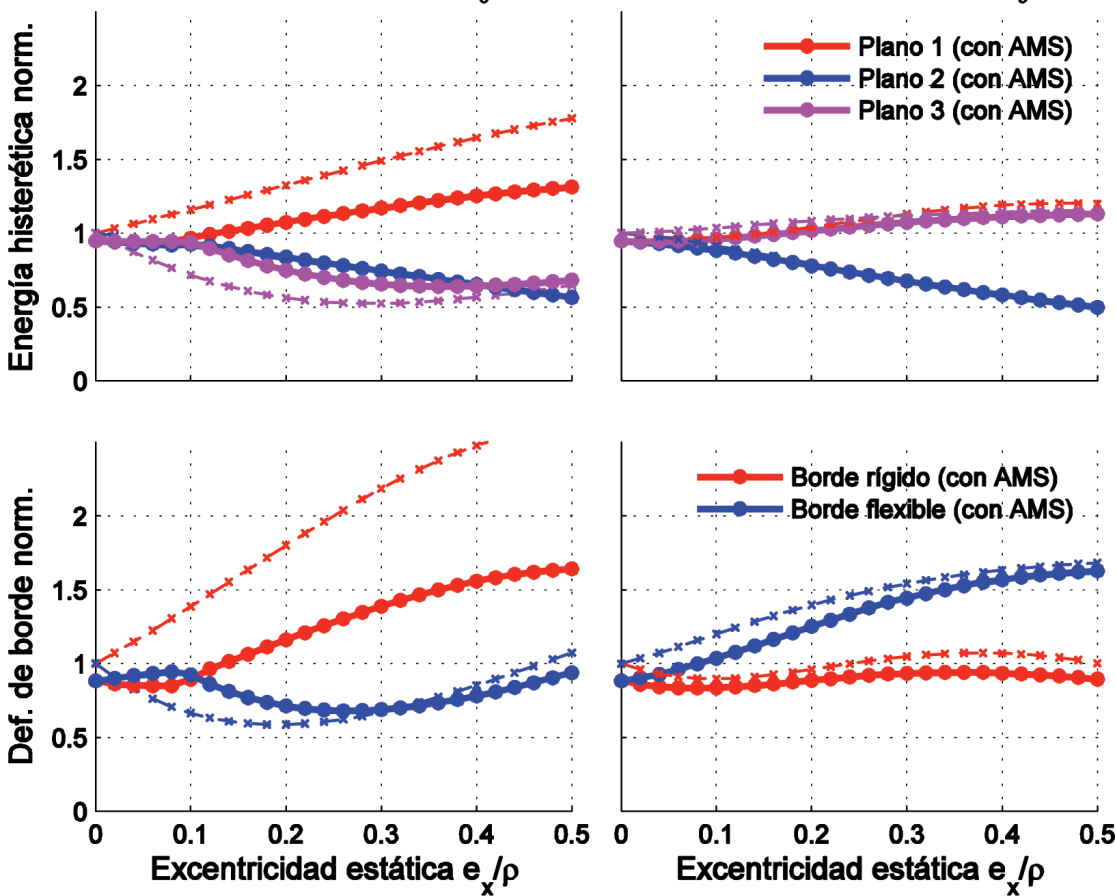

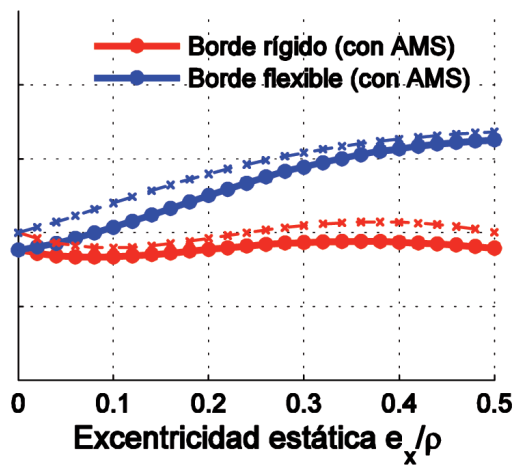

Figura 10: Energía y varianza de deformaciones de borde normalizadas según Criterio $1, \mathrm{AB}$ amplio $\left(T_{\mathrm{s}}=2 \mathrm{~s}, \xi_{\mathrm{s}}=0.05, \mu=0.02\right.$,

$R=5, n=1$ y $\alpha_{1}=\alpha_{2}=\alpha_{3}=0.5$ ).

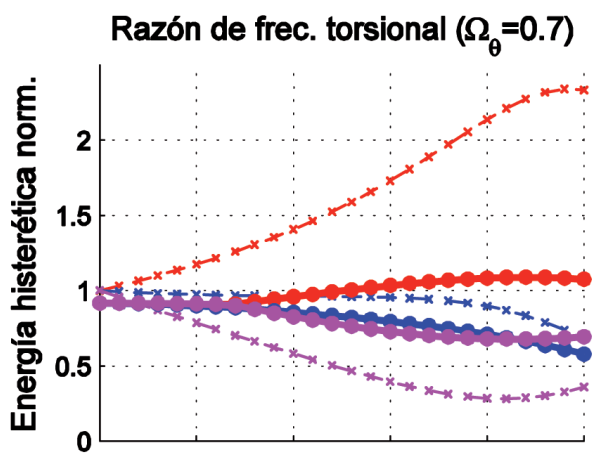

Razón de frec. torsional $\left(\Omega_{\theta}=1\right)$
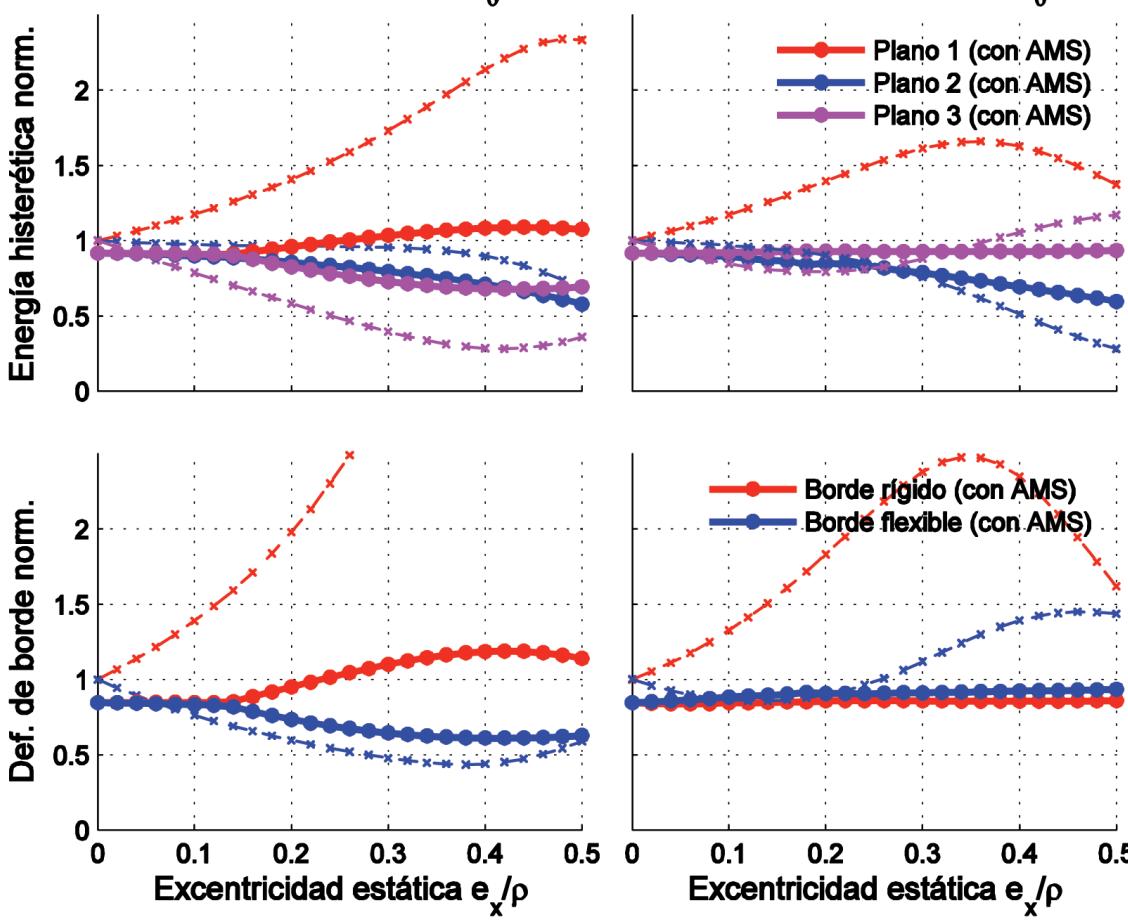

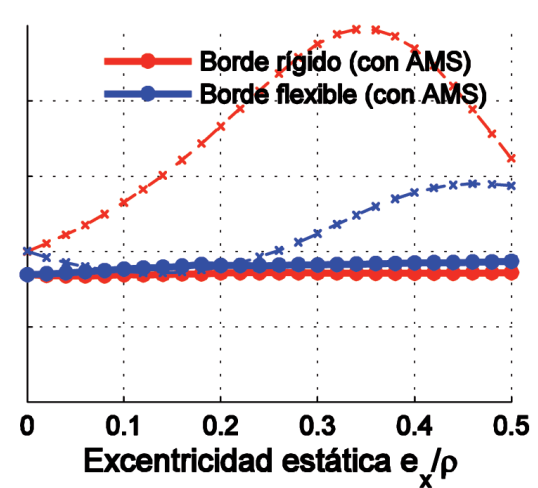

Razón de frec. torsional $\left(\Omega_{\theta}=1.3\right)$
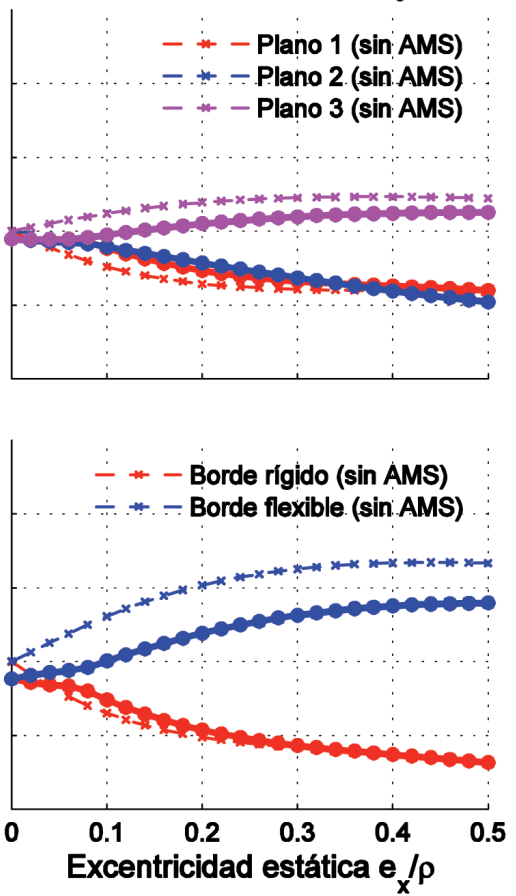

Figura 11: Energía y varianza de deformaciones de borde normalizadas según Criterio $1, \mathrm{AB}$ angosto $T_{\mathrm{s}}=2 \mathrm{~s}, \xi_{\mathrm{s}}=0.05, \mu=0.02, R$ $=5, n=1$ y $\alpha_{1}=\alpha_{2}=\alpha_{3}=0.5$ ). 

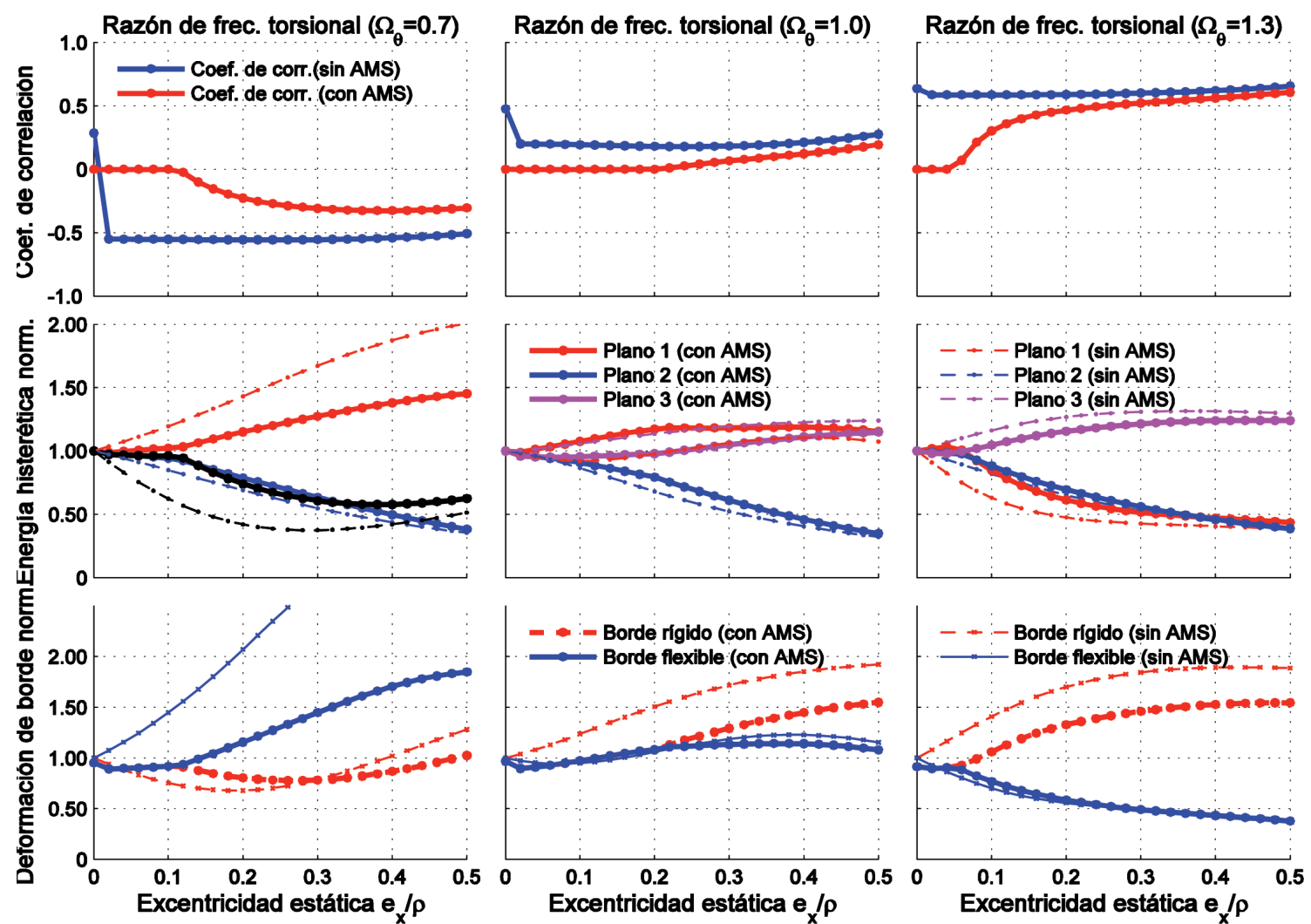

Figura 12: Coeficiente de correlación, razón de energía histerética y razón de desviación estándar de deformaciones de borde de la estructura. Proceso de AB amplio según Criterio $2\left(T_{\mathrm{s}}=2 \mathrm{~s}, \xi_{\mathrm{s}}=0.05, \mu=0.02, R=5, n=1\right.$ y $\left.\alpha_{1}=\alpha_{2}=\alpha_{3}=0.5\right)$
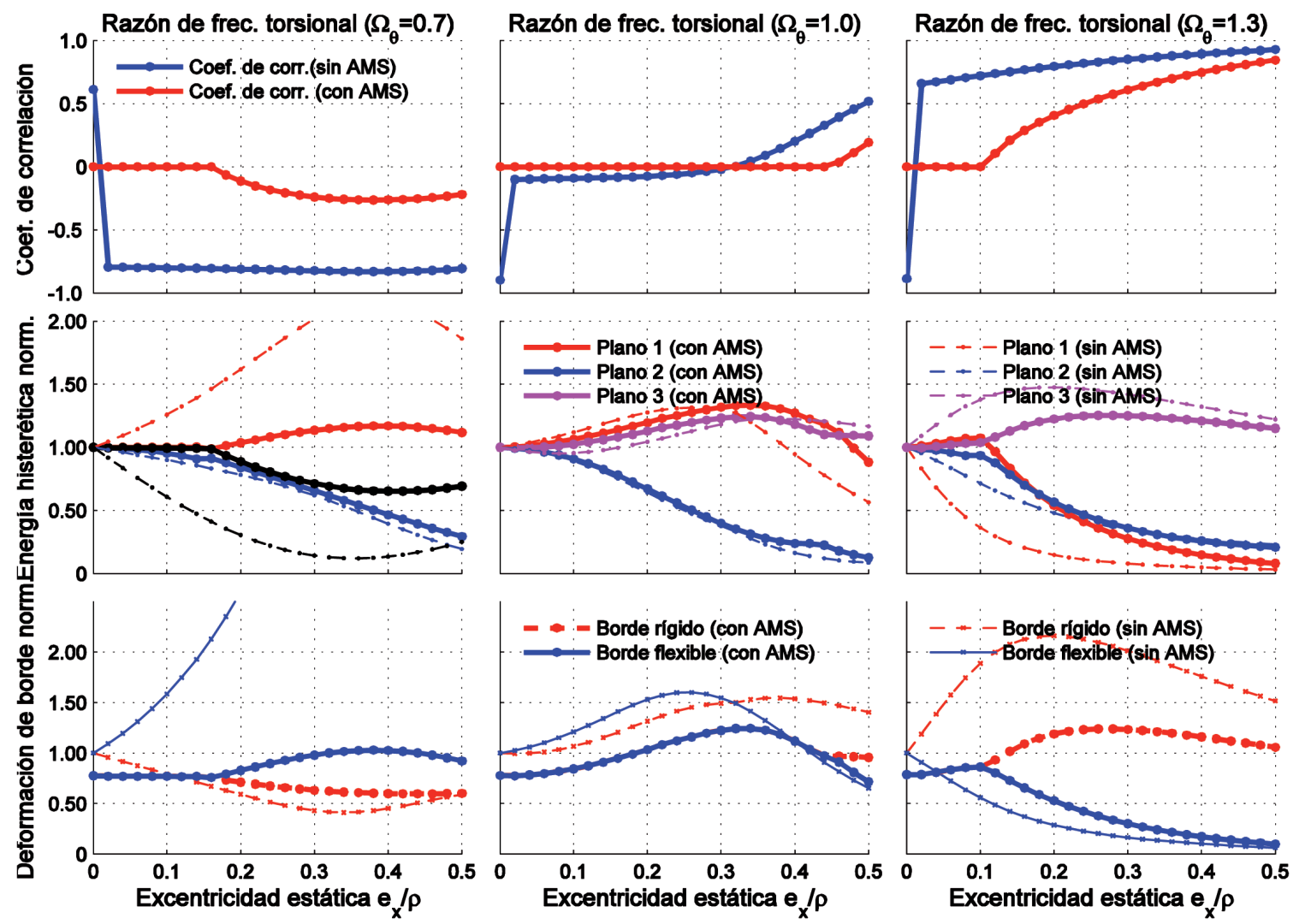

Figura 13: Coeficiente de correlación, razón de energía histerética y razón de desviación estándar de deformaciones de borde de la estructura. Proceso de AB angosto según Criterio $2\left(T_{\mathrm{s}}=2 \mathrm{~s}, \xi_{\mathrm{s}}=0.05, \mu=0.02, R=5, n=1\right.$ y $\left.\alpha_{1}=\alpha_{2}=\alpha_{3}=0.5\right)$ 
La Figura 13 muestra el coeficiente de correlación $\rho_{u \theta}$ del sistema con respecto a la excentricidad normalizada de la planta, la energía histerética normalizada de cada plano resistente, con y sin presencia de AMS en el sistema y la desviación estándar de deformación normalizada de los bordes rígidos y flexibles para la misma estructura de la Figura 12 para un proceso de $\mathrm{AB}$ angosto, la distribución de ellos es análoga a la Figura 12. Se observa un comportamiento similar para la energía histerética, produciéndose un balance de energía histerética de los tres ejes, pero en un rango de excentricidades mayor. Por otra parte, la inclusión del AMS en la estructura produce balance torsional en un rango de excentricidades mayor que en el caso de $\mathrm{AB}$ amplio, alcanzando casi todo el rango de excentricidades para estructuras torsionalmente híbridas.

Las Figuras 14 y 15 muestran las razones de energía y desviación estándar de los bordes con AMS con respecto a la misma estructura sin AMS, con el objetivo de analizar la eficiencia del AMS. Las propiedades de la estructuras son $T_{s}=2 \mathrm{~s}, \xi_{s}=0.05, \mu=0.02, R=5, n=1 \quad \mathrm{y} \quad \alpha_{1}=\alpha_{2}=\alpha_{3}=$ 0.5 ) y está sometida a un proceso de $\mathrm{AB}$ amplio (Figura 14) $\mathrm{y}$ a un proceso de $\mathrm{AB}$ angosto (Figura 15). En la primera fila se entregan las razones de energía histerética de los tres planos no lineales y en la segunda las razones de la desviación estándar de desplazamiento para el borde rígido y el borde flexible. Se observa que tanto en procesos de $\mathrm{AB}$ amplio, como de $\mathrm{AB}$ angosto, en el eje en que se ubica el AMS es donde se obtiene la mayor reducción de energía disipada, mientras en los demás amplifica. Se observa que en todos los casos la mayor amplificación o reducción se produce para el criterio 2 . Se observa también que en estructuras torsionalmente híbridas sometidas a procesos de $\mathrm{AB}$ amplio prácticamente no hay reducción ni tampoco amplificación de la razón de desplazamiento. Para el proceso de $\mathrm{AB}$ angosto el aumento de energía disipada es considerablemente mayor, ya que el comportamiento de la estructura sin AMS muestra una distribución muy desigual de la energía disipada por cada plano resistente, lo que se traduce en planos con valores de energía disipada muy pequeños, que producen valores de razón de energía disipada muy altos. Se observa también que las reducciones y amplificaciones son mayores en el caso de excitaciones de $\mathrm{AB}$ angosto, en especial para el criterio 2.
Razón de frec. torsional $\left(\Omega_{\theta}=0.7\right)$
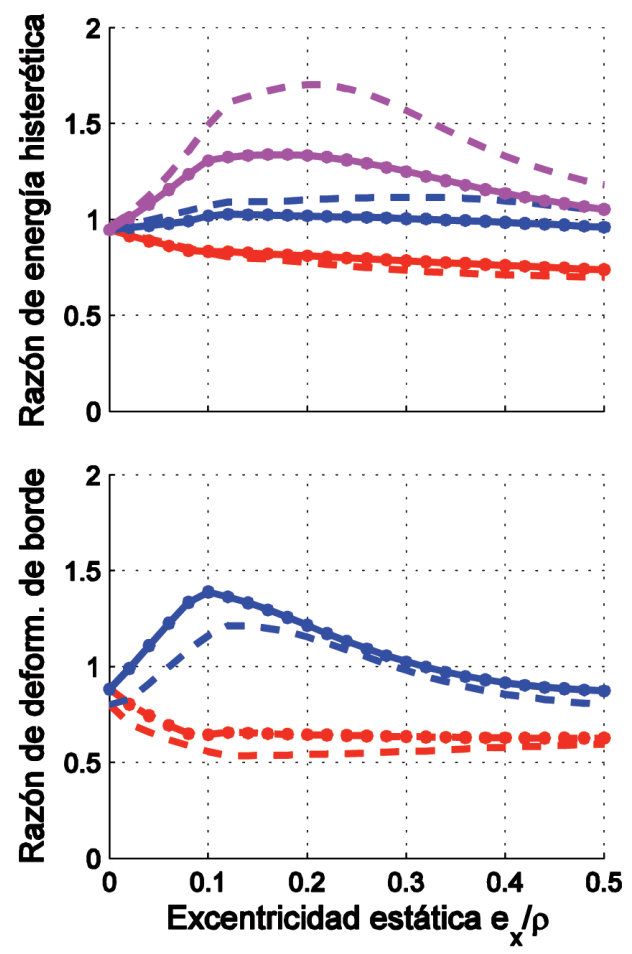

Razón de frec. torsional $\left(\Omega_{\theta}=1.0\right)$
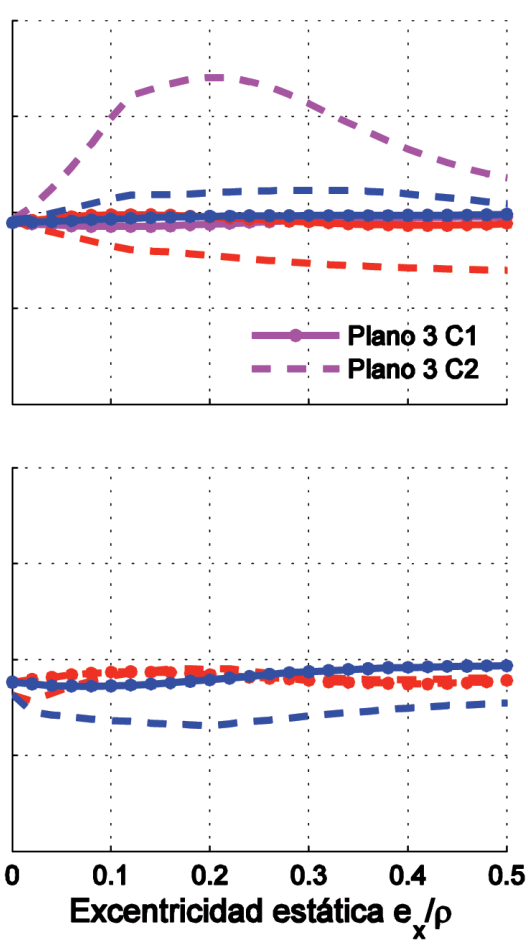

Razón de frec. torsional $\left(\Omega_{\theta}=1.3\right)$
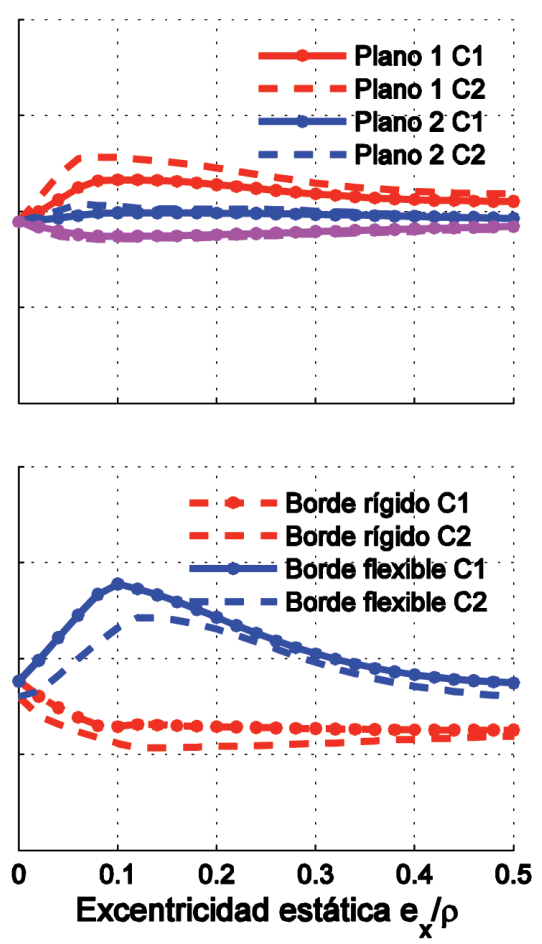

Figura 14: Razón de energía y varianza de deformaciones de borde, AB amplio ( $\left.{ }^{\mathrm{c}} A M S / \mathrm{s} A M S\right)\left(T_{\mathrm{s}}=2 \mathrm{~s}, \xi_{\mathrm{s}}=0.05, \mu=0.02, R=5\right.$, $n=1$ y $\alpha_{1}=\alpha_{2}=\alpha_{3}=0.5$ ) 

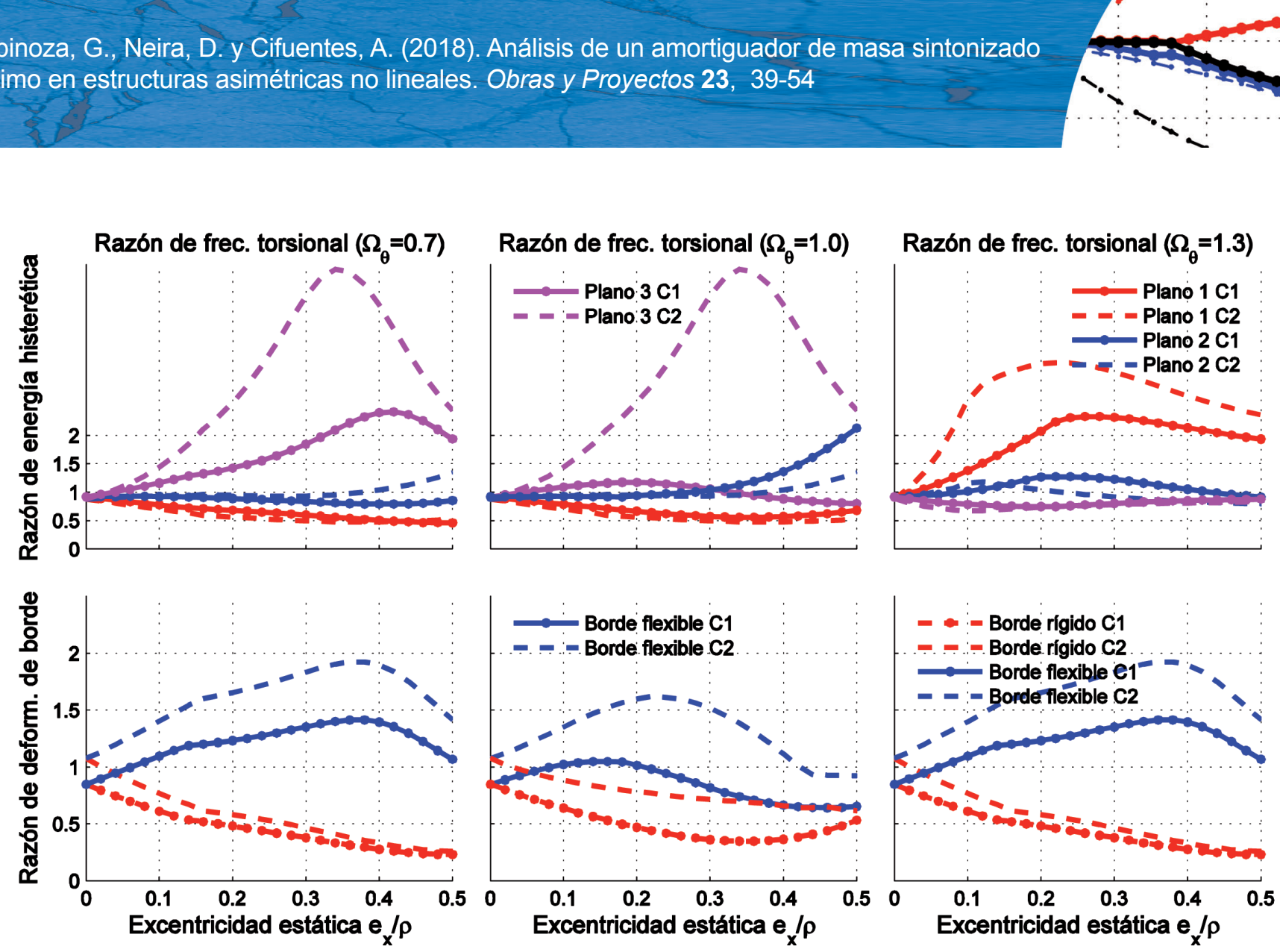

Figura 15: Razón de energía y varianza de deformaciones de borde, $\mathrm{AB}$ angosto ( $\left.{ }^{\mathrm{c}} A M S / \mathrm{s} A M S\right)\left(T_{\mathrm{s}}=2 \mathrm{~s}, \xi_{\mathrm{s}}=0.05, \mu=0.02, R=5\right.$, $n=1 \quad$ y $\left.\alpha_{1}=\alpha_{2}=\alpha_{3}=0.5\right)$

\section{Conclusiones}

De esta investigación se puede concluir que ambos criterios de optimización son muy sensibles tanto a la posición como a la frecuencia del AMS. Sin embargo, se observa una insensibilidad de los funcionales con respecto al amortiguamiento del AMS, por lo que este parámetro no afecta significativamente en la respuesta óptima del sistema. En cuanto al comportamiento de los parámetros óptimos del AMS para ambos criterios, se observó que para el caso de AB amplio, la frecuencia del AMS tiende a sintonizarse con la frecuencia lineal equivalente del modo predominante, mientras para el caso de $\mathrm{AB}$ angosto, el AMS tiende a sintonizarse con la frecuencia dominante de la excitación sísmica. La posición óptima del AMS tiende a situarse en el borde que presenta mayor daño con la estructura sin AMS, en los criterios utilizados. En ambos criterios para procesos de $\mathrm{AB}$ amplio, a medida que la estructura incursiona más en el rango no lineal, el AMS se va desintonizando con respecto a la frecuencia óptima del caso lineal, mientras que en procesos de $\mathrm{AB}$ angosto, la frecuencia óptima es independiente del grado de no linealidad de la estructura. Sólo se logra balancear el daño en los ejes resistentes de la estructura para valores de excentricidad bajos (en promedio $e_{s} / \rho<0.1$ ) hasta que la posición del AMS llega al borde de la planta, para los dos criterios. La implementación del AMS en estructuras asimétricas no lineales logra reducir la energía disipada, del sistema asimétrico con respecto al sistema no controlado asimétrico, del eje resistente más cercano a la ubicación del dispositivo, provocando una amplificación en los ejes restantes.

\section{Agradecimientos}

Esta investigación ha sido financiada por la Universidad del Bío-Bío a través de Proyecto Regular de Investigación DIUBB 086414 2/R. Los autores agradecen el apoyo recibido. También los autores agradecen al Dr. José Luis Almazán de la Pontificia Universidad Católica de Chile, al Dr. Patricio Álvarez y al Dr. Álvaro Suazo de la Universidad del Bío-Bío por su colaboración en la revisión del manuscrito. 


\section{Referencias}

Almazán, J.L., Espinoza, G. and Aguirre, J.J. (2012). Torsional balance of asymmetric structures by means of tuned mass dampers. Engineering Structures 42, 308-328

Baber, T.T. and Wen, Y.K. (1981). Random vibration hysteretic, degrading systems. Journal of the Engineering Mechanics Division 107(6), 1069-1087

Benavente-Climent, A., Morillas, L. and Escolano-Margarit, D. (2014). Inelastic torsional seismic response of nominally symmetric reinforced concrete frame structures: Shaking table tests. Engineering Structures 80, 109-117

Bouc, R. (1967). Forced vibration of mechanical systems with hysteresis. Fourth Conference on Nonlinear Oscillation, Prague, Czechoslovakia

Bouc, R. (1969). Modèle mathématique d'hystérésis et application aux systèmes à un degré de liberté. Thése Sc. Phys., University d'Aix-Marseille, France (en Francés)

Clough, R.W. and Penzien, J. (1975). Dynamics of structures. $2^{\text {nd }}$ edition, McGraw-Hill

Den Hartog, J. P. (1947). Mechanical vibrations. McGraw-Hill

Jangid, R.S. and Datta, T.K. (1997). Performance of multiple tuned mass dampers for torsionally couple system. Earthquake Engineering and Structural Dynamics 26(3), 307-317

Kwok, K.C.S. and Samali, B. (1995). Performance of tuned mass dampers under wind loads. Engineering Structures 17(9), 655-667

Lin, C.C., Ueng, J.M. and Huang, T.C. (2000). Seismic response reduction of irregular buildings using pasive tuned mass dampers. Engineering Structures 22(5), 513-524

Lin, J.L., Wang, W.C. and Tsai, K.C. (2016). Suitability of using the torsional amplification factor to amplify accidental torsion. Engineering Structures 127, 1-17
NCh2745 (2003). Análisis y diseño de edificios con aislación sísmica. Instituto Nacional de Normalización INN, Santiago, Chile

Sgobba, S. and Marano, G.C. (2010). Optimum design of linear tuned mass dampers for structures with nonlinear behaviour. Mechanical Systems and Signal Processing 26(6), 1739-1755

Singh, M.P., Singh, S. and Moreschi, L.M. (2002). Tuned mass dampers for response control of torsional buildings. Earthquake Engineering and Structural Dynamics 31(4), 749-769

Soto-Brito, R. and Ruiz, S. (1999). Influence of ground motion intensity on the effectiveness of tuned mass dampers. Earthquake Engineering and Structural Dynamics 28(11), 1255-1271

Ueng, J.M., Lin, C.C. and Wang, J.F. (2008). Practical design issues of tuned mass dampers for torsionally coupled buildings under earthquake loadings. The Structural Desing of Tall and Special Buildings 17(1), 133-165

Villaverde, R. (1994). Seismic control of structures with damped resonant appendages. $1^{\text {st }}$ World Conference on Structural Control, Los Angeles, California, USA, vol. 1, 113-122

Wen, Y.K. (1976). Method for random vibration of hysteretic systems. Journal of the Engineering Mechanics Division 102(2), 249-263

Wong, K.K. (2008). Seismic energy dissipation of inelastic structures with tuned mass dampers. Journal of Engineering Mechanics 134(2), 163-172

Zhang, Z. and Balendra, T. (2013). Passive control of bilinear hysteretic structures by tuned mass damper for narrow band seismic motions. Engineering Structures 54, 103-111 